811.131.1'26

https://doi.org/10.18485/italbg.2018.1.1

\author{
Michele Rainone* \\ Università degli Studi di Roma "La Sapienza"
}

\title{
SU UNA QUERELA PER STUPRO DEL 1770: NORMA GRAMMATICALE, LINGUA D'USO E CONDIZIONAMENTI TESTUALI IN ALCUNI DOCUMENTI BUROCRATICI DI AREA PUGLIESE**
}

\begin{abstract}
Il contributo è basato sull'analisi morfo-sintattica e testuale di una querela depositata nel 1770 per uno stupro commesso nella Terra di Monteleone in provincia di Foggia. Il testo rappresenta in modo adeguato alcune delle caratteristiche linguistiche rilevanti di un corpus ben più ampio costituito da trenta fascicoli processuali attualmente raccolti nella "serie IX" del fondo "Dogana delle pecore" dell'Archivio provinciale. Seguendo gli sviluppi più recenti delle ricerche linguistiche in chiave diacronica, l'analisi si sofferma non solo sul rapporto tra singoli fenomeni, norma grammaticale e usi coevi, ma anche sulla relazione intercorrente fra usi linguistici e tipologia testuale. Lo scopo è evidenziare che la specificità di tali scritture consiste nella compresenza di forme e usi di segno opposto, da una parte ascrivibili alla lingua della burocrazia; dall'altra all'italiano dei semicolti.

Parole chiave: italiano burocratico, italiano dei semicolti, lingua d'uso nel Settecento, querela per stupro, Regia Dogana della mena delle pecore, usi linguistici e tipologia testuale.
\end{abstract}

\section{COORDINATE STORICO-GEOGRAFICHE E PRESENTAZIONE DEL CORPUS}

L'Archivio di Stato di Foggia conserva nella "serie IX" del fondo dedicato alla "Regia Dogana della mena delle pecore" un migliaio di atti processuali riguardanti i reati commessi nell'ultimo trentennio del Sette-

*michele.rainone@outlook.com

** Ringrazio Grazia Battista dell'Archivio di Stato di Foggia, Adriana Di Biase e Francesco Bianco per i preziosi consigli e suggerimenti. Naturalmente la responsabilità di quanto affermato resta di chi scrive. 
cento e nei primi anni dell'Ottocento dai cittadini un tempo sottoposti alla giurisdizione del Tribunale doganale.

La Dogana fu istituita da Alfonso V d'Aragona con una prammatica del primo agosto 1447 per regolamentare la transumanza tra 1'Abruzzo e la Puglia all'interno dell'imponente sistema dei tratturi, i sentieri deputati al pascolo delle greggi, e fu soppressa con una legge del 21 maggio 1806 promulgata da Giuseppe Bonaparte. Il Tribunale fu fondato in séguito durante il regno di Ferrante I d'Aragona ed esercitò il suo controllo su una rete di province estesa nelle regioni dell'Abruzzo, del Molise, della Puglia, della Campania e della Basilicata ${ }^{1}$.

Il corpus di riferimento di questo lavoro è costituito da trenta fascicoli processuali ${ }^{2}$ relativi ai reati verificatisi soltanto nei centri minori e maggiori

${ }^{1}$ Non è possibile tuttavia tracciare con precisione i confini dell'area soprattutto a causa della perdita di documenti dovuta al terremoto del 1731 e al secondo conflitto mondiale; è certo però che il sistema dei tratturi comprendesse le seguenti province: L'Aquila, Chieti, Pescara, Teramo, Campobasso, Isernia, Foggia, Bari, Brindisi, Taranto, Lecce, Avellino, Benevento, Potenza e Matera. Riguardo alle riproduzioni cartografiche una delle più complete consiste in una carta di P. di Cicco ed E. Caruso poi integrata da M. C. Nardella e S. Russo: un importante lavoro di «semplificazione di una geografia di terre e diritti molto complessa e articolata» (d'Atri e Russo 2008: 27-31). Sulle dimensioni dell'area e sulla storia della Dogana cfr. Palasciano (1984) e bibliografia ivi indicata.

${ }^{2}$ Ogni fascicolo è indicato con un numero romano a partire da I; le forme sono citate con i numeri arabi del foglio e del rigo in cui compaiono (i fogli bianchi non sono considerati ai fini della numerazione). Di séguito si riportano le informazioni principali sui fascicoli, cioè il numero identificativo, la data indicata sulla copertina, i comuni di provenienza e residenza degli interrogati (che non sempre coincidono), e tra parentesi la collocazione nel fondo: I: 1770 - Cagnano, Carpino, Monte Sant'Angelo, San Giovanni Rotondo (7, 161); II: 1773 - Ascoli Satriano, Terra di Aschi (Ortona dei Marsi, L'Aquila) (37, 719); III: 1770 - Lucera (7, 159); IV: 1782 - Alberona, San Bartolomeo in Galdo (75, 1415); V: 1770 - Foggia (7, 160); VI: 1773 - Foggia, Ugento (37, 717); VII: 1770 - Foggia $(7,158)$; VIII: 1771 - Foggia, Roma, Torremaggiore (7, 142); IX: 1782 -Faeto, Greci (75, 1413); X: 1782 - Foggia, Montepeloso (oggi Irsina) (75, 1425); XI: 1784 - Rodi, San Marco in Lamis (100, 1796); XII: 1771 - San Marco in Lamis, Terra di Costarella (San Severino, Salerno) (7, 152); XIII: 1784 - Foggia (100, 1784); XIV: 1773 - Alberona, Castellammare, Santa Maria in Vulgano, Terra di Biccari $(37,725)$; XV: 1771 - Acquaviva di Bari, Castellaneta, Foggia, Pietramontecorvino (7, 139); XVI: 1782 - Casalnuovo, San Paolo, San Severo (75, 1412); XVII: 1782 - Foggia (75, 1410); XVIII: 1782 -Foggia, Roccaraso (75, 1411); XIX: 1773 - Foggia (37, 728); XX: 1773 - Foggia (41, 795); XXI: 1782 - Apricena, Foggia (75, 1418); XXII: 1773 - Corato, Foggia, Pietra (forse Pietramontecorvino) (37, 723); XXIII: 1771 - Lucera, San Severo (7, 148); XXIV: 1773 - Carpino, Cerignola, Foggia, Ischitella, Torremaggiore (37, 714); XXV: 1770 - San Severo (1, 5); XXVI: 1770 - Castelluccio Vallemaggiore, Montefalcone, Roseto, Stornara (1, 1); XXVII: 1784 - Ariano, Ascoli Satriano, Bisaccia, Candida, Foggia (101, 1802); XXVIII: 1770 - Foggia $(2,24)$; 
della Capitanata, e fornisce preziose informazioni sulla lingua in uso nelle varie sezioni territoriali del Tribunale. Più precisamente la specificità di tali scritture si deve alla coesistenza di usi, forme e costrutti ascrivibili da una parte all'italiano burocratico, collocabile tra le varietà scritte, formali e socialmente alte (Berruto 2010: 19-27); dall'altra al continuum delle varietà semicolte $^{3}$ e più in generale alle varietà substandard.

Si registrano dunque fenomeni marcati gli uni in alto in diafasia gli altri in basso in diastratia, nonché in diatopia, i primi dovuti alla specificità della lingua burocratica; i secondi all'evidente «alfabetizzazione asimmetrica» (Biasci 2004: 156) degli scrivani: con tale definizione Gianluca Biasci descrive il profilo linguistico di suor Maria Leonarda, scrivente che non rientra di certo tra i professionisti della scrittura come avrebbero dovuto esserlo al contrario gli scrivani della Dogana; anche nei testi prodotti da questi ultimi emerge tuttavia che «porzioni della norma sufficientemente padroneggiate si giustappongono a porzioni più indigeste, in parte o per nulla assimilate» (ibidem). Sebbene le prammatiche insomma individuino nello scrivano un «idoneo, e buono scrittore» (di Stefano 1731: 520, § 24), nei fascicoli non si rinvengono soltanto gli usi collocabili nel solco della tradizione burocratica: sono presenti infatti sia forme e costrutti che riproducono i fenomeni dell'area centro-meridionale e dei dialetti ${ }^{4}$ sia usi non marcati diatopicamente ma comunque devianti dalla norma grammaticale coeva.

Gli esempi commentati in questa sede intendono sottolineare da una parte come la tradizione e la prassi burocratica abbiano influenzato la scrittura dello scrivano, dalla scelta di singole forme sino all'organizzazione complessiva del testo; dall'altra come oltre agli usi ascrivibili alle varietà substandard siano rappresentati anche costrutti e forme sì devianti dalla norma ma che, ricorrendo nelle produzioni degli scriventi più istruiti, non possono essere classificati come substandard tout court.

XXIX: 1770 - Monteleone (1, 12); XXX: 1771 - Altamura, Andria, Cerignola, Gravina, Pisticci, Tolve, Valenzano (12, 263).

${ }^{3}$ Gli studi più recenti tendono a considerare quella di semicolto come nozione «scalare» (Bianco e Špička 2017: 21): di qui la necessità di una «ridistribuzione graduale, lungo un continuum, delle competenze scrittorie di coloro che producono i testi, troppo spesso accorpati [...] in un'unica categoria indistinta» (Fresu 2014: 200).

${ }^{4}$ Per i fenomeni areali cfr. almeno Avolio (1995) e bibliografia ivi indicata. Sulle forme dialettali e più in generale sul rapporto tra oralità e scrittura nei fascicoli processuali cfr. Rainone (in stampa). 


\section{LE SCRITTURE PROCESSUALI TRA NORMA GRAMMATICALE, DEVIAZIONI E LINGUA D'USO ${ }^{5}$}

\subsection{La rilevanza dei verbali e delle deposizioni ai fini dell'analisi linguistica}

I fascicoli processuali sono costituiti in genere da un verbale posto in apertura, seguito dalle deposizioni delle parti coinvolte nel processo, dei testimoni citati e di esperti interpellati a vario titolo, ad esempio di dottori se il reato commesso consiste in una ferita o in un omicidio, nonché dalle comunicazioni ufficiali con cui si ordina di fare qualcosa, quasi sempre di recarsi presso la sede principale della Dogana: si tratta perciò di testi tipologicamente diversi poiché i verbali e le deposizioni hanno una funzione essenzialmente narrativo-descrittiva ${ }^{6}$; gli atti ufficiali sono invece testi prescrittivi.

Quest'analisi è basata sui primi tipi di testo: limitarsi agli atti ufficiali significherebbe restituire infatti un'immagine parziale del corpus essendo tali comunicazioni evidentemente esemplate su modelli preesistenti che permettono solo in minima parte di analizzare gli usi dello scrivano. Si vedano le tre comunicazioni che seguono, redatte rispettivamente da Nicola Maria Mosca, Raffaele d'Aloij e Leonardo Barrusso ${ }^{7}$ :

(1) Appartiene al servizio del Reg(i)o Fisco aversi da noi le | qui sotto annotate persone di q(ue)sta Cittá di Lucera, e com- | moranti in essa. Per tanto abbiamo stimato fare | il p(resen)te, con cui loro dicemo, ed ordinamo che subito post abbiano, e debbano, e ciascuno di esse abbia, e debba di per- | sona conferirsi avanti di noi in casa di n(ost)ra Resid(enz)a, perché| informati saremo di q(ua)nto ci fa di bisogno sapere saran- $\mid$ no con tutta la brevitá possibile licenziati. Tanto dun- | que eseguano, e non altrimenti, per q(ua)nto tengono

${ }^{5}$ I testi sono trascritti senza ammodernare l'uso dei segni d'interpunzione; il carattere maiuscolo e l'univerbazione sono riprodotti inoltre solo per i casi indubbi. La barra verticale singola | e la barra verticale doppia || indicano la prima le interruzioni di riga, la seconda il cambio di pagina; le parentesi ( ) sono usate invece per lo scioglimento delle abbreviazioni.

${ }^{6}$ La funzione descrittiva prevale nelle relazioni di perizia degli esperti: le deposizioni dei dottori consistono ad esempio in lunghe e precise descrizioni di ferite o più in generale dello stato di salute del paziente.

${ }^{7}$ Le firme apposte alla fine degli atti sono tuttavia due: nel primo compare anche quella di Diegantonio D'Argentio; nel terzo quella di Pasquale Maselli. Nel secondo compaiono invece le firme di Francesco Nicola de Dominicis e Saverio Malenconico, e non quella del presunto scrivano d'Aloij. Solo confrontando il ductus delle firme e quello dei testi anche successivi si è risaliti all'ufficiale che ha redatto la comunicazione. 
cara | la Grazia Reg(i)a, e sotto pena di oncie d'oro venticinque | Fisco Reg(i)o (per) per ciascheduno controveniente. Il p(rese)nte Lu- | cera li 31: Agosto 1770: (III, 19, 6-17)

(2) Incumbendo (per) servizio del Reg(io) Fisco aversi da noi le $\mid$ qui dietro annotate persone, abbiamo perció spedito il $\mid \mathrm{p}$ (rese)nte, col quale le dicemo ed ordinamo, che subito post si conferiscano avanti di noi in q(ue)sta Reg(i)a Doana, per- | che informati saremo di quel tanto ci necessita sa- $\mid$ pere, saranno licenziate. Si guardino del contrario - | se stimano cara la Gra(zia) Reg(ia) e sotto pena d'once d'oro | venticinque (per) ciasched(un)o [...] li $\underline{7}$ Giugno 1773 (XXII, 21, 5-12)

(3) Giurati. Necessitando per serviggio di questa Principal | Corte le dietro annotate persone di questa T(err)a di | Monteleone; a quali farete ordine, e mand(a)to sotto $\mid$ pena d'oncie d'oro 25. (per) ciascuno Controve(nien)te [...] si conferiscono avanti di noi, che in | formati saremo di quanto ci necessita sapere $\mid$ sarando disbrigate. Tanto eseguano, e non al | trim(ent)i, [...] Dato in | Monteleone lì 9. Gennajo 1770 (XXIX, 12, 4-12)

Si considerino le forme serviggio e sarando nel brano (3): la prima riproduce la geminazione intervocalica dell'affricata prepalatale sonora; la seconda invece è con tutta probabilità una forma ipercorretta di saranno per reazione all'assimilazione -ND- $>-n n$ - tipica dei dialetti centro-meridionali. Tali forme sono tra i pochi casi registrati in documenti di questo genere che testimoniano l'influenza esercitata dall'oralità sul testo scritto e che più in generale permettono di analizzare la reale competenza dello scrivano: per il resto gli atti sono caratterizzati con evidenza da una ripetitività che riguarda non solo i contenuti ma anche le risorse linguisticotestuali impiegate.

Si veda anzitutto la struttura stessa dell'atto, costituito da un'iniziale parte espositiva in cui si motiva l'invio della comunicazione, da una parte prescrittiva in cui si ordina di fare qualcosa e da una parte finale in cui tale ordine viene ribadito. Si notino in secondo luogo le dittologie sinonimiche dicemo ed ordinamo che sono tra le figure retoriche più ricorrenti nel corpus e più in generale tra quelle ben attestate nella prosa burocratica, degli «sterili pleonasmi» secondo Addeo (1939): tra gli altri se ne trovano esempi anche in testi tipologicamente affini di area veneziana (Tomasin 2001: 84).

Gli atti riprodotti peraltro sono pressoché simili a quelli di tutto il corpus: esistono dunque uno o più modelli di riferimento memorizzati o copiati direttamente dallo scrivano che non favoriscono un'analisi linguistica articolata. Anche i verbali e le deposizioni sono influenzati da modelli 
preesistenti e risultano caratterizzati dalla reiterazione di formule e moduli fissi: in questo caso tuttavia lo scrivano si trova a ricostruire meticolosamente reati di volta in volta diversi e ciò favorisce un'analisi che tenga conto anche di usi non dipendenti da un modello comune. Per queste ragioni e soprattutto per la presenza nel testo di un numero di usi, forme e costrutti tale da rappresentare adeguatamente l'intero corpus si è ritenuto opportuno incentrare il lavoro sul commento della querela che segue, depositata nel 1770 da Grazia Tabasso della Terra di Monteleone nella provincia di Foggia per una violenza subita nel $1769^{8}$ :

(4) Grazia Tabasso di questa T(er)ra di Monteleone, dice esse- | re vergine in Capillis ${ }^{9}$ figlia del fù mastro Gior- | gio Tabasso, e Petronilla Lescio di questa sud(dett)a $\mid \mathrm{T}(\mathrm{er}) \mathrm{ra}$, d'età sua d'anni diecinnove in c(irc)a, ut dixit, | principalis cu(m) juram(en)to int(errogat)a, et exam(ina)ta, et p(ri)mo.

Int(errogat)a come si trova essa principal Deponente avanti $\mid$ del d(ett)o Sig(no)r Gov(ernator)e, dixit; Essendo io stata stuprata | da Giovanni di Antonio Casullo di questa sud(dett)a $\mid \mathrm{T}$ (er)ra sotto promessa di Matrim(oni)o, e non vedendo attesa | la promessa fattami di sposare, perciò mi sono por | tata avanti di V(os)S(ignoria) (per) farlo astringere col mezzo della $\mid$ Giustizia $^{10}$.

E dettoli come passa il fatto dello stupro commesso in perso- $\mid$ na di essa principal deposante dal d(ett)o Giovanni | Casullo, quando, dove, ed in che modo. dixit $\operatorname{Sig}($ no)re abi=| tando di Casa nell'luogo $^{11} \mathrm{~d}(\mathrm{ett}) \mathrm{o}$ la strada de Bovierii | vicino la Casa di Cristofano Volpe mio Paesano | solitaria senza nessuno in Casa (per) essere povera | orfana $^{12}$ senza Padre, e Madre, sulli principij | del passato mese di

${ }^{8}$ Anche in questo testo (cfr. n. prec.) si legge la firma di Pasquale Maselli ma lo scrivano è Leonardo Barrusso. Sono necessarie alcune precisazioni sui criteri di trascrizione del testo: l'accento è indicato dall'ufficiale o con un punto virgolettato o con un semplice punto, oppure con un tratto perpendicolare alla tonica; nei primi due modi è indicato anche il punto di $i$ minuscolo: ciò comporta l'impossibilità di stabilire se le parole tronche in -ì (cosi, sì ecc.) siano state accentate o meno. Si è ritenuto d'intervenire dunque usando l'accento grave o acuto per le tronche (normalizzando l'accentazione di quelle in - $i$ ) e il punto per $i$ minuscolo. Per una migliore leggibilità le sezioni principali del testo sono state separate.

${ }^{9}$ Cioè non ancora sposata.

${ }^{10}$ Attestata nella $4{ }^{\mathrm{a}}$ Crusca (s.v. astrignere e astringere), nell'Ottocento il Dizionario del Petrocchi considera tale espressione in disuso.

${ }^{11}$ L'apostrofo è indicato con un punto, come accade anche successivamente in son 'io son'io.

${ }^{12}$ L'impiego del costrutto per + infinito presente con valore causale è ben attestato nel corpus (si noterà peraltro come con il passato rientri nell'uso contemporaneo). Numerose 
Marzo del caduto anno 1769, | non ricordandomi il preciso giorno (per) la lunghezza $\mid$ del tempo, cominciò il sud(dett)o Giovanni Casullo ad $\mathrm{amo}=\mid$ reggiare meco, con venire continuatam(ent)e in d(ett)a $\mid$ mia Casa, e facendomi promessa di volermi $\mid$ sposare, con tale occasione io lo corrispondeva $\|$ agli amoreggiam(ent)i (per) la promessa sud(dett)a, e ciò durò conti | nuatam(ent)e per tutti j giorni de mesi trascorsi dal d(ett)o mese di Marzo, sino al prossimo passato mese di $\mid$ Agosto, sulla fine poi dello sud(dett)o mese d'Agosto co- | minciò il sud(dett)o Giovanni à cercarmi dispiacere, di- | cendo / Statemi meco, perche io ti voglio sposare; ${ }^{13}$ | Io a tali parole non volli acconsentire, dicendoli | che era uccisa, e maltrattata da miej parenti, | il sud(dett)o ciò non volle sentire, ma perche stava infuria- $\mid$ to, mi prese, e mi buttò vicino al mio letto, che in d(ett)a mia | Casa tenea, ed alzandomi la gonnella a forza, $\mathrm{po}=\mid$ se mano al suo membro, e me l'appuntò nelle mie $\mid$ coscie, propriam(ent)e nelle parti vergognose d'avanti ${ }^{14}$, | e perche io non volea acconsentire, lo stesso Gio(vanni) | molto si affatigò, senza che mi avesse potuto | rompere, ma cogli sfrecam(en)ti, che lo stesso facea $\mid$ si corruppe, a motivo che io intesi in $\mathrm{d}(\mathrm{ett}) \mathrm{e}$ mie | coscie una cosa calda che mi bagnò al quanto, e $\mid$ così mi lasciò, e se n'andiede, e non venne in $\mathrm{d}(\mathrm{ett}) \mathrm{a} \mid$ mia Casa per lo spazio di giorni quindeci, il gior- $\mid$ no susseguente à medesimi, tornò di nuovo in d(ett)a $\mid$ mia Casa, ed avendomi ritrovata anche sola, fece | il medesimo atto, che pocanzi vi hò narrato corrum- | pendosi il medesimo, senza che mi avesse rotta, mà $\mid$ tal atto di sfrecam(ent)o lo facea dentro le d(ett)e mie | coscie, con baciarmi, e fare altri atti che a lui | piacevano; Nella mettà poi del mese di Settembre del d(ett)o || del d(ett)o scorso anno 1769, da sopra, o sotto, non ricordan | domi il giorno preciso (per) la lunghezza del tempo, in | un giorno sopravenne di nuovo il pred(ett)o Giovanni | verso La mettà della notte, ed aprendomi La mia | finestra, la quale stà poco alta da sopra la terra, | entrò (per) la medesima in $\mathrm{d}(\mathrm{ett}) \mathrm{a}$

attestazioni sono anche in alcune lettere familiari di primo Ottocento scritte da mittenti cólti (Antonelli 2003: 179-180). Un caso con l'infinito diverso da essere (per vedersi rotto) è attestato nell'esempio (9).

${ }^{13}$ Oltre ad essere tratteggiato, il discorso diretto è introdotto da una barra tondeggiante leggermente obliqua, qui riprodotta in modo stilizzato: potrebbe trattarsi di una virgula antica, in passato usata anche con tale funzione (Coluccia 2008: 84). Si noti che in chiusura ricorre il punto e virgola. Nel testo è presente un altro esempio, ma con una virgula posta in chiusura: / io ti levo la stima, ed io te la rendo / (XXIX, 3, 16).

${ }^{14} \mathrm{Il}$ riferimento è ai genitali (l'accezione è registrata anche nella $4^{\mathrm{a}}$ Crusca, s.v. parte). 
mia Casa, e trovò a me | che stava in letto dormendo, io avendo inteso tal $\mathrm{ru}=\mid$ more, ed avendo veduta, ed inteso una persona, mi | presi grandiss(i)mo timore, ed avendomi dato parola, | dicendo son'io son'io ${ }^{15}$, e così avendolo conosciuto, che $\mid$ era il sud(dett)o Giovanni m'acchetai; Il med(esi)mo perche $\mid$ stava in camiscia, e calzonetti, si venne a colcare $\mid$ nell'letto dove io solitaria dormiva, e cominciando- $\mid$ mi a baciare, si pose sopra di me, e perche io non | volea acconsentirlo, il med(esim)o mi diceva queste simili | parole / io ti levo la stima, ed io te la rendo /, ed | altre persuasive, e promesse, che mi faceva, in ma- $\mid$ niera che m'indusse, che io l'acconsentij, ed avendosi | posto mano al suo membro virile, mi alzò la cami- | scia, e voltatami alla supina in d(ett)o mio letto, mi | appuntò il d(ett)o suo membro indurito nella bocca della | mia natura $^{16}$, e nel spingere, e rispingere che fece $\mid$ lo stesso Giovanni, intesi io cadere dentro della $\mid \mathrm{d}(\mathrm{ett}) \mathrm{a}$ mia natura una cosa come fusse acqua te- $\mid$ pida, e standosi (per) altre poche ore in d(ett)o mio letto, | discorrendo, alla fine poi verso due ore mattino || si alzò, e se n'andiede via; Essendomi alzata io la | mattina al far del giorno da letto, mi accorsi, e vid $\mid$ di, che nella mia camiscia vi stavono dalla parte $\mid$ di dietro alcune macchie di sangue, e così poi segui $=\mid$ tò da giorno in giorno $\mathrm{d}(\mathrm{ett}) \mathrm{a}$ copula, venendosene il | d(ett)o Giovanni sempre di notte a colcarsi con me in d(ett)a | mia Casa, tanto vero che fin dal mese d'Ottobre ver $=\mid$ so la fine del med(esim)o non mi venne il mio Solito mese | e così mi son veduta gravida, come di presente $\mathrm{so}=\mid$ no, e così passa il fatto, ed è la verità.

E dettole se delle cose pred(ett)e ne fa querela, dixit Sì sig(nor)e, | e domando che sia astretto d(ett)o Giovanni a sposar- | mi come mi hà promesso

E dettoli chi può deponere le cose sud(dett)e dixit Carobina | Gurriero, Agnesa Scarano, Pietro Miscia, Saba= | to Tollo, Rocco Lallone, Tomaso la Manna, Ma- | scia Giusep(p)a Annicchiarico, Carminella la Moglie | di Giusep(p)e Lombardo, ed altri $\operatorname{Sig}(\mathrm{nu}) \mathrm{m}$ Crucis (XXIX, 1, 2-4, 19)

${ }^{15} \mathrm{Il}$ discorso diretto non è aperto da alcun segno di punteggiatura, a differenza dei casi commentati alla n. 13.

${ }^{16} \mathrm{Il}$ riferimento è agli organi genitali femminili (l'accezione è registrata anche nella $4^{\text {a }}$ Crusca). 


\subsection{Condizionamenti testuali e aspetti morfo-sintattici rilevanti della lingua burocratica}

A livello morfologico e sintattico-testuale la querela riprodotta è caratterizzata dalla presenza di usi specifici della prosa burocratica, alcuni dei quali già riscontrabili in testi due-trecenteschi (al riguardo cfr. almeno le sintesi di Mortara Garavelli 2001 e Lubello 2017).

Si noti anzitutto la rigidità della struttura testuale: la divisione in più sezioni che caratterizza l'atto si ripresenta pressoché sistematicamente in tutte le deposizioni del corpus. Tale struttura è costituita da una sezione in cui viene descritto minuziosamente il reato preceduta da una sezione in cui si citano le generalità dell'interrogato e da un'altra in cui viene dichiarato il motivo per cui ci si è recati presso la sede della Dogana. Dopo la sezione narrativa sono presenti altre due sezioni: una in cui viene chiesto esplicitamente se si intenda sporgere querela e una in cui si chiede di citare eventuali testimoni. La maggior parte delle sezioni è inoltre aperta da una proposizione retta da un verbo di domanda (Interrogata, dettole / dettoli), cui fa séguito una reggente con dixit (in altri atti respondit). È evidente insomma che lo scrivano sia stato influenzato da un modello di riferimento preesistente.

Una certa formularità caratterizza anche la parte strettamente narrativa: non ricordandomi il preciso giorno (per) la lunghezza | del tempo è un modulo attestato (seppur con varianti diverse) in altri fascicoli; l'uso di prossimo passato in sino al prossimo passato mese di $\mid$ Agosto è inoltre un burocratismo, peraltro registrato anche in alcuni testi coevi di semicolti ${ }^{17}$.

Tra gli aspetti più rilevanti a livello testuale si segnala l'ipercoesione dovuta al frequente uso di attributi deittico-anaforici (il suddetto e il predetto Giovanni ecc.) che possono ricorrere in coreferenza con i possessivi (detta mia casa, dette mie coscie, detto mio letto ecc.), con i dimostrativi (questa suddetta Terra) e con sintagmi aventi funzione di specificazione (detto mese di Marzo). Gli esempi (5), (6) e (7) mostrano come la compresenza di elementi linguistici anaforici possa generare una certa ridondanza informativa: in (5) essa dipende dalla compresenza del dimostrativo e della relativa. In (6) è il periodo lungo e complesso a favorire un duplice rinvio (con il dimostrativo il medesimo) allo stesso referente (Giovanni Casullo) creando una catena anaforica piuttosto pesante. In (7) invece la ridondanza dipende dall'uso contestuale di un pronome personale che indica un referente nella sua totalità $(m e)$ e di un possessivo che ne indica solo una parte (mie coscie). Si noti peraltro che dai primi due esempi si evince non tanto la

${ }^{17}$ Ad esempio nelle memorie difensive scritte da Giovanni Garbino durante la detenzione (Testa 2014: 66-72). 
scarsa competenza dello scrivano quanto la necessità di eliminare qualsiasi traccia di ambiguità referenziale:

(5) il medesimo atto, che pocanzi vi hò narrato (XXIX, 2, 23)

(6) Il med(esi)mo perche I stava in camiscia, e calzonetti, si venne a colcare $\mid$ nell'letto dove io solitaria dormiva, e cominciando- $\mid \mathrm{mi}$ a baciare, si pose sopra di me, e perche io non | volea acconsentirlo, il med(esim)o mi diceva queste simili | parole (XXIX, 3, 11-6)

(7) me L'appunto nelle mie coscie (XXIX, 2, 12-3)

Il testo è visibilmente influenzato dalla prassi scrittoria degli ufficiali quando questi possono spingersi fino alla copiatura di interi periodi per velocizzare il disbrigo delle pratiche; si vedano ad esempio i seguenti stralci tratti dalle deposizioni di Carmina Miscia (8) e Caterina Pezzullo (9), levatrici di Monteleone interpellate per verificare lo stato di salute di Grazia Tabasso dopo lo stupro:

(8) Ed avendo colla porta / chiusa, e colla finestra aperta di d(ett)a Casa fatta | coricare sopra il letto d(ett)a vergine Grazia Tabasso, | e scovertole le sue parti vergognose d'avanti, | ed osservata la sua natura, avemo visto, come hò | veduto, osservato, e riconosciuto, che d(ett)a Grazia | e Stuprata, per vedersi rotto il suo pannicolo || verginale, tanto che avendo io, e d(ett)a Caterina Pezzullo | una doppo l'altra posto due dita unite dentro | d(ett)a sua natura sono entrate senza alcuna resisten- $\mid$ za. qual sud(dett)o Stupro apparisce fatto da più tem- $\mid$ po, ed avemo giudicato, come giudico, che sia stato $\mid$ causato da membro virile, eretto (XXIX, 8, 18 - 9, 6)

(9) Ed avendo colla $\mid$ porta chiusa, e colla finestra aperta di d(ett)a casa fatta $\mid$ coricare sopra il letto d(ett)a vergine Grazia Tabasso, e | scovertole le sue parti vergognose d'avanti, ed | osservata la sua natura, avemo visto, come hò vedu- | to, osservato, e riconosciuto, che d(ett)a Grazia è Stu- | prata, per vedersi rotto il suo pannicolo verginale, | tanto che avendo io, e d(ett)a Carmina Miscia una dopo | l'altra posto due dita unite dentro d(ett)a sua natu- | ra, sono entrate senza alcuna resistenza. qual | sud(dett)o Stupro apparisce fatto da più tempo, ed $\|$ avemo giudicato, come giudico che sia stato causato da $\mid$ membro virile eretto (XXIX, 10, 17 - 11, 2)

Entrambe le deposizioni sono state redatte da Leonardo Barrusso e sono pressoché identiche l'una all'altra. Casi di questo tipo non rappresentano delle eccezioni e potrebbero dipendere non solo dalla necessità di velocizzare il lavoro di trascrizione ma anche dall'intenzione di ridurre al minimo, se non eliminare del tutto, le differenze tra le perizie degli esperti. 
La presenza di latinismi e di costrutti dal sapore libresco, aulico o arcaizzante è senza dubbio un altro aspetto che i testi del corpus condividono con la prosa burocratica.

Si noti anzitutto la topologia latineggiante nella deposizione (4): 1'ordine dei costituenti frasali è caratterizzato da inversioni tra soggetto e verbo (cominciò il suddetto Giovanni Casullo) oppure tra quest'ultimo e oggetto diretto (il suddetto ciò non volle sentire) o altri sintagmi (che in detta casa tenea). È uno degli aspetti sintattico-testuali più evidenti assieme all'ipercoesione e alla rigida organizzazione dei contenuti.

Latineggianti sono anche altri costrutti, ad esempio le infinitive con soggetto proprio (10) e la negazione con i verba timendi o con perifrasi aventi la medesima sfumatura semantica (11):

(10) che perciò la sud(dett) a Vacca si diceva essere sta- | ta rubbata, e scorticata (I, 70, 21-2); d(ett) a ferita, giudicammo $\mid$ subito [...] essere stata causata da Istrum(en)to | appontuto (V, 21, 4-6); ed ora stimo Io di essere tal rossore, | e segni stato causato da percossa di mano aperta (XIII, 33, 5-6); si erano rotti due mattoni, che pretendea essere rifatti (XIII, 51, 5-6); ed al lustrare dell'aria, co- | nobbi, bene essere Giuseppe Meladanti (XV, 23, 17-8)

(11) ma sul dubbio di non ricevere Io qualche in- | contro, e per vivere quieto, stimai succhiudere la porta (XV, 25, 15-6); cosi temen- | do di non essere [...] offeso [...] l'ho chiamato in aggiuto (XIX, 7, 5-7); e dubbitando che il sud(det) to $\operatorname{Sam}(\mathrm{m})$ arco non avves $=\mid$ se fatto qualche innovaz(io)ne nel muro (XXIII, 8, 22-3); cosicche essa mia figlia $\mid$ temendo di non essere offesa [...] si era nascosta (XXIV, 88, 5-7)

Rientrano nel gruppo delle forme ricercate e arcaizzanti gli infiniti che in (4) sono preceduti da con aventi sfumatura modale (con venire, con baciarmi) e che in altri testi si aggiungono a quelli preceduti da in (12): pur essendo frequente nel Seicento (Migliorini 1987: 427), il costrutto preposizionale era raro già nel primo Ottocento $(i v i, 569)^{18}$ ed è considerato libresco e probabilmente arcaizzante nella seconda metà del secolo (Serianni 1990: 87).

(12) e questi in vederla, li tolse [...] d(ett)a mazza (VII, 2, 12-3); ed il Romaniello in cio' sentire disse (X, 19, 27-8); in sentire la Giovanna $\mid$ nominare la dilei figlia [...] si approssimó alla Chiara (XIII, 53, 6-8); non assisteva in conformita' della sua obligazione | in custodire i Forni assignatoli (XV, 19, 25-6)

${ }^{18}$ Sono numerose tuttavia le attestazioni segnalate da Antonelli (2003) nel suo studio sulle lettere familiari scritte da mittenti cólti. 
Nelle deposizioni (8) e (9) è attestato inoltre un indicativo presente in -emo, avemo, séguito peraltro da una terna costruita con i verbi vedere, osservare e riconoscere ${ }^{19}$ : nel secolo XVIII forme di questo genere sono ritenute antiche-avemo lo è ad esempio per il Gigli (1729: 72) - e in alcuni casi «pur da guardarsene» (Soave 1802: 71). Esse trovano senz'altro terreno fertile nella prosa burocratica, anche se il Dembsher nel suo Manuale, o sia guida per migliorare lo stile di cancelleria invita ad essere accorti nell'uso: «Il tuo buon giudizio ti permetta, alcuna volta far risorgere quelle antiche maniere» (Lubello 2016: 55).

L'influenza della tipologia testuale, quindi il condizionamento del tipo di testo sugli usi dello scrivano, è ben evidente nei casi in cui questi ricorre a quale senza articolo, una delle risorse con funzione coesiva più impiegate nel corpus, sia come semplice pronome (13) sia come nesso relativo (14) ${ }^{20}$ :

(13) ivi rattrovò ${ }^{21}$ tredeci pezzi di Cojo Vaccina ${ }^{22}$, | quali furono presi, e consignati (I, 20, 14-5); venne deposto l'ac | capo della Informaz(io)ne, quale addossata ad un [...] Subalter | no, sono state acquistate le seguenti pruove (VIII, 3, 4-6) ${ }^{23}$; si viddero [...] violentata, ed aperta la porta [...] ed assalita da Fran(cesco) $\mid$ Paolo Ortone, e Fran(cesco) Paolo Labriola [...], quali entrarono accompagnati da un'altra persona (XXIV, 19, 12-5)

(14) teneva ${ }^{24}$ una $\mid$ ferita nella sua testa [...] qual feri- | ta è lunga due dita e mezzo (II, 6, 23-7); ne feci ricorso con | ist(anz)a formale [...] $q$ (ua)le istanza [...] domando, che mi sia mostrata, e $\mid$ letta (III, 9, 16 - 10, 3); ne feci due mie giurate | deposiz(io)ni [...] quali deposiz(io)ni [...] domando che mi si mo- $\mid$ strano (XVI, $42,14-20)$

${ }^{19} \mathrm{Si}$ noti al riguardo che il medesimo verbo è flesso inizialmente alla prima persona plurale (avemo visto), successivamente alla prima persona singolare (ho veduto). Nello stesso brano è attestato un altro esempio di tale figura retorica: avemo giudicato, come giudico.

${ }^{20}$ Sul costrutto cfr. la sintesi e la bibliografia in Bianco (2009) che per sottolineare il valore aggettivale del relativo preferisce usare la definizione di «aggettivo relativo».

${ }^{21}$ Nei fascicoli è ben rappresentata la forma rattrovare 'ritrovare', non lemmatizzata tuttavia nel Vocabolario della Crusca e nel Dizionario del Petrocchi. Alcune indicazioni sulla connotazione sociolinguistica della forma vengono da alcune pubblicazioni ottocentesche, per le quali soltanto ritrovare «non Rattrovare è nel Vocabolario» (Ferracci 1824: 83); rattrovare rappresenta infatti un «pessimo allungamento di trovare» (Ugolini 1859, s.v. rattrovare).

22 'Cuoio di bovino' (di una vacca rubata da alcuni ladri e in séguito scorticata).

${ }^{23}$ Si noti non soltanto la frattura sintattica tra quale e il resto del periodo ma anche la forma non fiorentina pruove.

${ }^{24}$ La selezione di tenere in luogo di avere per esprimere il possesso è un tratto marcato arealmente ed è pressoché sistematico nel corpus (cfr. almeno Rohlfs 1969: § 733; Telmon 1994: 617-618). 
La norma coeva lo considera un «abuso de' volgari» (Corticelli 1754: 79). Tale tendenza non è attestata tuttavia solo nelle scritture semicolte, ad esempio nelle memorie di Giovan Francesco Fongi (Testa 2014: 65), nelle Lettere di suor Maria Crostarosa (Librandi e Valerio 1996: 72, 81, 108, passim) e in quelle di suor Maria Leonarda (Biasci 2004: 147): se ne trovano esempi infatti anche nella lingua di autori come il Gravina, il Vico, il Giannone e il Visconti (ibidem).

Ciò che interessa maggiormente in questa sede è sottolineare come l'uso fosse diffusissimo nella lingua burocratica del Quattrocento (Palermo 1994: 179-84) e che appartiene senz'altro alle «forme bandiera della produzione cancelleresca» (Lubello 2017: 49): si tratta di una sorta di forma inerziale ${ }^{25}$ memorizzata dallo scrivano in questo modo per questi testi. È evidente insomma che esista una stretta correlazione tra un certo uso linguistico e la tipologia testuale di riferimento: considerare substandard quale privo di articolo significherebbe commettere di conseguenza un errore di prospettiva e offrire un'analisi solo parziale del dato.

I casi sinora discussi permettono senz'altro di collocare questi testi nell'àmbito delle scritture burocratiche; tali esempi non sono tuttavia sufficienti per tracciare un quadro completo dei reali usi dello scrivano: sono attestati infatti altrettanti casi che esemplificano come la norma sia stata interiorizzata solo parzialmente e che quindi, pur tenendo conto della specificità della singola forma e del singolo uso, sono ascrivibili alle varietà basse degli assi di variazione diastratico e diafasico.

\subsection{Deviazioni dalla norma: casi significativi di fenomeni areali e lingua d'uso}

Nella deposizione (4) si rinvengono ad esempio fenomeni di area centro-meridionale: si considerino la forma camiscia 'camicia' in cui è riprodotto l'esito -SJ- > S (Avolio 1995: 69), l'oggetto preposizionale in trovò a me

${ }^{25}$ Con il concetto di «aulicismo d'inerzia» sono stati indicati alcuni aulicismi della lingua poetica, quelli di routine, «usati al di qua di una vera intenzione stilistica, come uno strumentario neutro attinto alla koinè scritta dell'epoca» (Mengaldo 1987: 228). Il concetto ben si adatta a questo caso. 
| che stava in letto dormendo ${ }^{26}$ e l'uso di stare in luogo di essere (Telmon 1994: $617-8)^{27}$ avente sia valore copulativo (15) sia valore locativo (16):

(15) ma perche stava infuria- | to (XXIX, 2, 9-10)

(16) nella mia camiscia vi stavono [...] alcune macchie di sangue (XXIX, 4, 3-4)

La forma di imperfetto stavono dell'esempio (16) non è ammessa peraltro nei testi normativi sette-ottocenteschi (sul tipo cfr. la coniugazione di amare in Gigli 1729: 82 e quelle di amare, temere, credere e sentire in Mastrofini 1814: 48, 55, 61, 66).

A testimonianza della scarsa o non del tutto compiuta interiorizzazione della norma da parte dello scrivano è interessante osservare come in alcuni casi questi faccia ricorso a due pronomi di cortesia diversi per lo stesso referente: in Statemi meco, perche io ti voglio sposare (XXIX, $2,6)$ ad esempio viene usato all'inizio il pronome di seconda persona plurale, com'è tipico del Meridione (Rohlfs 1968: § 477), poi quello di seconda singolare.

Che la norma fosse stata scarsamente interiorizzata dagli scrivani è testimoniato anche dal tasso di oscillazione elevato che caratterizza la lingua dei documenti. Si consideri la trascrizione degli antroponimi: nella deposizione (4) il cognome della querelante è Tabasso ma tra gli altri nella copertina del fascicolo si legge anche Tabbasso con geminazione della bilabiale in posizione intervocalica. Tale alternanza non è un caso isolato: Carobina Gurriero è in realtà Cherubina Guerriero, come si legge in XXIX,

${ }^{26}$ In una frase con sequenza SVO e oggetto diretto caratterizzato dal tratto [+ umano] ed espresso o da un sostantivo o da un pronome il costrutto è senz'altro tipico delle varietà centro-meridionali. In altri contesti però, ad esempio con particolari verbi o nel caso di oggetto diretto dislocato a destra o sinistra, il costrutto ha una diffusione areale maggiore; nel caso delle dislocazioni a sinistra è persino panitaliano (almeno con certi tipi di verbo o espressioni) ed è attestato peraltro anche in varietà non popolari né regionali. La bibliografia sull'argomento è vastissima: per la diffusione areale del costrutto cfr. almeno Rohlfs (1969: § 632) e Avolio (1995: 94-95); per la presenza in italiano settentrionale cfr. Berretta (1991); una sintesi orientativa è in D'Achille (2006: 183). Riguardo ai secoli XVIII e XIX si noti inoltre che nella $4^{\text {a }}$ Crusca e nel Dizionario del Petrocchi è attestato solo l'uso transitivo di trovare.

${ }^{27} \mathrm{Si}$ noti però che il verbo fa parte dei regionalismi «rimasti vivi nel parlato-parlato» (Serianni 2004: 65) ed è attestato anche in scrittori settentrionali poiché «nei valori locativi la contiguità semantica tra i due verbi [essere e stare] è notevole» (Serianni 2009: $§ 138$ ). Non tutti gli usi oggi marcati in diatopia lo erano nel secolo XVIII: nella $4{ }^{\text {a }}$ Crusca (s. $v$. stare) si legge ad esempio stare confuso, stare contento, stare pensoso ecc. 
20, 2; nel testo I è inoltre attestata l'oscillazione tra le forme Scanzuso e Scansuso ${ }^{28}$.

L'oscillazione non riguarda soltanto la resa grafica degli antroponimi: in (17) ad esempio ricorrono con funzione copulativa prima il verbo essere poi il verbo stare; in (18) sono usate due forme analogiche dello stesso verbo a distanza di poche righe: lo scrivano usa sia andorono sia andiedero che, invise alle grammatiche coeve ${ }^{29}$, sono però ben attestate nel corpus (andiede peraltro è presente anche nella deposizione di Grazia Tabasso):

(17) non volli acconsentire, dicendoli | che era uccisa, e maltrattata [...] | il sud(dett)o ciò non volle sentire, ma perche stava infuria- | to mi [...] mi buttò vicino al mio letto (XXIX, 2, 7-10)

(18) el'andorono à cer- | care che volevano un paro di Scanate $^{30}$ di pane, e [...] le dissi, che fussero ritorna- | te la sera seguente, [...] come in | fatti sene andiedero [...] e tornorono poi | à venire (I, $56,17-22)$

Pur non essendo diatopicamente connotati, alcuni usi mostrano comunque quanto l'oralità abbia influenzato il testo scritto: si considerino i casi di ridondanza pronominale (19) e quelli di dislocazione (20), attestati peraltro non solo nelle scritture semicolte: le dislocazioni ad esempio sono numerose nelle lettere di mittenti cólti studiate da Antonelli (2003: 211-214).

(19) facendomi promessa di volermi $\mid$ sposare (XXIX, 1, 25-6)

(20) avendolo conosciuto, che $\mid$ era il sud(dett)o Giovanni (XXIX, 3, 10)

Per alcune forme devianti dalla norma è necessario adottare un approccio che contempli non solo il riscontro con le grammatiche ma che consideri anche una variabile come l'uso: si pensi al clitico $l i$ in dicendoli (21) e alla forma verbale viddi (XXIX, 4, 2-3).

(21) co- | minciò [...] Giovanni à cercarmi dispiacere, di- | cendo [...] Io a tali parole non volli acconsentire, dicendoli $\mid$ che era uccisa, e maltrattata da miej parenti (XXIX, 2, 4-8)

\footnotetext{
${ }^{28}$ L'emersione dei fenomeni fonetici nella grafia non sempre identifica delle scritture appartenenti all'italiano dei semicolti; sull'affricazione dopo nasale della sibilante cfr. ad esempio le considerazioni di Librandi (2004) che commenta alcune forme in Giambattista Vico in cui il fenomeno è riprodotto.

${ }^{29}$ Sui due tipi cfr. Rohlfs (1968: $\left.§ \S 568,579\right)$. I verbi in -orono (ad esempio affondorono) sono corretti in -arono e andiede è corretto in andò dal Gigli (1729: 118, 121); andiede è ritenuto incerto, erroneo anche dal Mastrofini (1814: 94-95), per il quale le forme in -orono «si riprovano» $(i v i, 51)$.

30 'Pagnotte'.
} 
Il primo ricorre in luogo di gli come oggetto indiretto. Niccolò Amenta (1724) sembra non condividere la posizione di chi considera corretto tale scambio, e infatti non lo ammette nella sua grammatica, anche se in séguito sottolinea di non biasimare chi assume una posizione diversa: «Tutti gli altri Grammatici pongon tra tali particelle [...] $l i$, per quelli, essi, o, a lui, ma noi non avendo voluto il $l i$ tra gli articoli nommen'il vogliam tra' Pronomi, e Vicepronomi, valendone della particella, gli, che lo stesso significa; non biasimando in tanto chi del, li, o sempre [...] valer si volesse» (ivi, 300). Difatti tra i grammatici vi è chi ritiene possibile l'uso di $l i$ obliquo maschile (Corticelli 1754: 61); lo scambio risulta attestato peraltro nell'epistolario volgare di Pietro Bembo (Prada 2000: 191-192). La prevalenza della forma li in luogo di gli e le in un corpus come questo di scritture medie e l'atteggiamento dei grammatici lasciano pensare insomma che il fenomeno sia ascrivibile all'uso del tempo e che non possa essere connotato pertanto come substandard tout court, tantomeno come dialettale (Berruto 1983: 47).

Le stesse considerazioni riguardano la forma viddi che, pur non essendo ritenuta corretta (cfr. tra gli altri Mastrofini 1814: 652), non è attestata solo nelle scritture semicolte, ad esempio nelle lettere di Amalia Ruspoli Pianciani al figlio (Poggiogalli 2004: 118), nella Cronaca teramana di Angelo De Jacobis (Fresu 2006: 156) e nelle Lettere di suor Maria Celeste Crostarosa (Librandi e Valerio 1996: 72, 89, 90, passim). Se ne trovano attestazioni infatti anche nelle lettere di scriventi istruiti (Carlo Antici, Monaldo Leopardi, Giuseppe Melchiorri, Gaetano Donizetti e Vincenzo Pianciani) studiate da Antonelli (2003: 156); non doveva trattarsi pertanto di una forma poco usata se si considera inoltre che nel corpus è di gran lunga più frequente rispetto all'alternativa con dentale scempia.

La casistica è decisamente ampia ma questi pochi esempi dovrebbero aver messo in evidenza la cifra caratteristica di tali documenti: i tratti marcati in alto in diafasia, in cui emerge «tutto l'italiano che è stato ben appreso» (Mioni 1983: 505-506), coesistono con forme marcate in basso non soltanto in diafasia, ma anche in diastratia che si inseriscono in un contesto caratterizzato da forti oscillazioni nell'uso, di cui non è sempre possibile comprendere la ratio.

\section{IL CORPUS COME CONTINUUM DI VARIETÀ BUROCRATICHE}

Mediante esempi tratti dal corpus e riferimenti ad altri testi coevi si è tentato di tracciare un quadro linguistico d'insieme delle produzioni degli

ufficiali della Regia Dogana di Foggia: è piuttosto evidente che gli scrivani non fossero dei semicolti e che avessero dimestichezza con la scrittura; so- 
prattutto con una certa tipologia di testo, quello burocratico, caratterizzato da tutta una serie di forme e costrutti consolidatisi nel tempo (cfr. supra il $\S 2.2$ ) e che almeno in parte ricorrono ancor oggi in documenti dello stesso genere (Lubello 2017).

È altrettanto evidente tuttavia come questi tratti coesistano con i fenomeni fonetici, morfo-sintattici e lessicali specifici delle scritture dei semicolti e dei registri meno formali (cfr. supra il $\S 2.3$ ), e che dipendano non soltanto dalla competenza del singolo scrivano, ma anche da altre variabili, ad esempio dal dialetto degli interrogati oppure dall'intenzione, più volte sottolineata, di velocizzare i processi di raccolta e trascrizione delle deposizioni.

Visti nella loro globalità, dunque, tali testi rientrano nel novero delle cosiddette «scritture non letterarie» (Serianni 2007: 13) non proponendosi fini d'arte ed essendo stati prodotti da scriventi sì alfabeti, ma senza una specifica educazione letteraria.

Restituirne un'immagine che non voglia essere parziale e che voglia invece tener conto delle loro diverse sfaccettature è possibile adottando un approccio che superi la generica dicotomia standard/substandard e che collochi i testi in un continuum di varietà burocratiche intercorrente tra un polo alto, caratterizzato dagli usi non devianti dalla norma grammaticale coeva e da quelli diafasicamente più formali e aulici, e un polo basso, caratterizzato invece dagli usi devianti che possono riprodurre i fenomeni tipici dell'area e dei registri meno controllati.

In tal senso il corpus va considerato non come un insieme indistinto di scritture ascrivibili sic et simpliciter alle varietà basse o alte degli assi di variazione diafasico e diastratico, bensì come un insieme di testi che, senz'altro accomunati da forme, usi e costrutti ricorrenti, devono la loro specificità soprattutto alle modalità con cui il singolo scrivano si è accostato alla scrittura e quindi a come ha appreso e interiorizzato la norma.

\section{BIBLIOGRAFIA}

Addeo, P. (1939). Lingua notarile. Lingua Nostra, 1, 47-51.

Amenta, N. (1724). Della lingua nobile d'Italia, e del modo di leggiadramente scrivere in essa, non che di perfettamente parlare (Vol. 2). Napoli: Antonio Muzio.

Antonelli, G. (2003). Tipologia linguistica del genere epistolare nel primo Ottocento. Sondaggi sulle lettere familiari di mittenti cólti. Roma: Edizioni dell'Ateneo.

Avolio, F. (1995). Bommèspra. Profilo linguistico dell'Italia centro-meridionale. San Severo: Gerni. 
Berretta, M. (1991). Note sulla sintassi dell'accusativo preposizionale in italiano. Linguistica, 31, 211-32.

Berruto, G. (1983). L'italiano popolare e la semplificazione linguistica. Vox Romanica, 42, 38-79.

Berruto, G. (2010). Sociolinguistica dell'italiano contemporaneo. Roma: Carocci.

Bianco, F. (2009). L'aggettivo relativo in italiano antico. Bollettino dell'Atlante Lessicale degli antichi volgari italiani, 2, 31-54.

Bianco, F. e Špička, J. (2017). Una domanda (ancora) aperta. In F. Bianco e J. Špička (a cura di), Perché scrivere? Motivazioni, scelte, risultati. Atti del convegno internazionale di studi (Olomouc, 27-28 marzo 2015) (pp. 13-28). Firenze: Cesati.

Biasci, G. (2004). Alfabetizzazione imperfetta: strategie interpuntive nelle lettere di suor Maria Leonarda. In G. Antonelli, C. Chiummo e M. Palermo (a cura di), La cultura epistolare nell'Ottocento. Sondaggi sulle lettere del CEOD (pp. 137-77). Roma: Bulzoni.

Coluccia, R. (2008). Teorie e pratiche interpuntive nei volgari d'Italia dalle origini alla metà del Quattrocento. In B. Mortara Garavelli (a cura di), Storia della punteggiatura in Europa (pp. 65-98). Roma-Bari: Editori Laterza.

Corticelli, S. (1754). Regole ed osservazioni della lingua toscana ridotte a metodo ed in tre libri distribuite. Bologna: Lelio dalla Volpe.

D’Achille, P. (2006). L'italiano contemporaneo. Bologna: il Mulino.

d'Atri, S. e Russo, S. (2008). Il Tavoliere e la transumanza. In S. Russo (a cura di), Sulle tracce della Dogana. Tra archivi e territorio (pp. 27-32). Foggia: Claudio Grenzi.

di Stefano, S. (1731). La Ragion Pastorale, over Comento sù la Pramatica LXXIX. de officio Procuratoris Caesaris (Vol. 2). Napoli: Domenico Roselli.

Ferracci, G. (1824). Compendioso corso filologico italiano ovver callilografia toscana divisa in due parti. Napoli: Avolio e Mirelli.

Fresu, R. (a cura di). (2006). La Cronaca teramana del canonico Angelo de Jacobis. Edizione critica con studio introduttivo e glossario. L'Aquila: Edizioni Libreria Colacchi.

Fresu, R. (2014). Scritture dei semicolti. In G. Antonelli, M. Motolese e L. Tomasin (a cura di), Storia dell'italiano scritto. Italiano dell'uso (Vol. 3, pp. 195-223). Roma: Carocci.

Gigli, G. (1729). Lezioni di lingua toscana. Venezia: Bartolomeo Giavarina.

Librandi, R. (2004). Varietà intermedie di italiano in testi preunitari. In R. Van Deyck, R. Sornicola e J. Kabatek (a cura di), La variabilité en langue. Langue parlée et langue écrite dans le présent et dans le passé (pp. 77-103). Gand: Communication \& Cognition. 
Librandi, R. e Valerio, A. (a cura di). (1996). Maria Celeste Crostarosa. Le Lettere. Materdomini: Editrice San Gerardo.

Lubello, S. (a cura di). (2016). Giuseppe Dembsher. Manuale, o sia guida per migliorare lo stile di cancelleria. Sesto Fiorentino: apice libri.

Lubello, S. (2017). La lingua del diritto e dell'amministrazione. Bologna: il Mulino.

Mastrofini, M. (1814). Teoria e prospetto ossia Dizionario critico de'verbi italiani coniugati specialmente degli anomali e malnoti nelle cadenze (Voll. 1-2). Roma: De Romanis.

Mengaldo, P. V. M. (1987). L'Epistolario di Nievo: un'analisi linguistica. Bologna: il Mulino.

Migliorini, B. (1987). Storia della lingua italiana. Milano: Bompiani/RCS Libri.

Mioni, A. M. (1983). Italiano tendenziale: osservazioni su alcuni aspetti della standardizzazione. In P. Benincà, M. Cortelazzo, A. L. Prosdocimi, L. Vanelli e A. Zamboni (a cura di), Scritti linguistici in onore di Giovan Battista Pellegrini (Vol. 1, pp. 495-517). Pisa: Pacini.

Mortara Garavelli, B. (2001). Le parole e la giustizia. Divagazioni grammaticali e retoriche sui testi giuridici italiani. Torino: Giulio Einaudi.

Palasciano, I. (1984). Le lunghe vie erbose (Tratturi e pastori nella Puglia di ieri). Lecce: Capone.

Palermo, M. (1994). Il Carteggio Vaianese (1537-39). Un contributo allo studio della lingua d'uso nel Cinquecento. Firenze: Accademia della Crusca.

Petrocchi, P. (1887-1891). Nòvo dizionàrio universale della lingua italiana (Voll. 1-2). Milano: Fratelli Treves.

Poggiogalli, D. (2004). Un esempio di italiano familiare di primo Ottocento: le lettere di Amalia Ruspoli Pianciani al figlio Luigi (1833-1839). In G. Antonelli, C. Chiummo e M. Palermo (a cura di), La cultura epistolare nell'Ottocento. Sondaggi sulle lettere del CEOD (pp. 95-135). Roma: Bulzoni.

Prada, M. (2000). La lingua dell'epistolario volgare di Pietro Bembo. Genova: Name.

Rainone, M. (in stampa). Fenomeni areali e tracce di parlato semi-spontaneo nelle scritture processuali della "Regia Dogana della mena delle pecore" di fine '700 in Capitanata. Italica Wratislaviensia, 9.

Rohlfs, G. (1968). Grammatica storica della lingua italiana e dei suoi dialetti. Morfologia (Vol. 2). Torino: Giulio Einaudi.

Rohlfs, G. (1969). Grammatica storica della lingua italiana e dei suoi dialetti. Sintassi e formazione delle parole (Vol. 3). Torino: Giulio Einaudi. 
Serianni, L. (1990). Il secondo Ottocento: dall'Unità alla prima guerra mondiale. Bologna: il Mulino.

Serianni, L. (2004). Gli epistolari ottocenteschi e la storia della lingua. In G. Antonelli, C. Chiummo e M. Palermo (a cura di), La cultura epistolare nell'Ottocento. Sondaggi sulle lettere del CEOD (pp. 51-65). Roma: Bulzoni.

Serianni, L. (2007). La storia della lingua italiana, oggi. Bollettino di italianistica, 2, 5-19.

Serianni, L. (2009). Italiano. Grammatica, sintassi, dubbi. Milano: Garzanti.

Soave, F. (1802). Grammatica ragionata della lingua italiana. Venezia: Paulo Santini.

Telmon, T. (1994). Gli italiani regionali contemporanei. In L. Serianni e P. Trifone (a cura di), Storia della lingua italiana. Le altre lingue (Vol. 3, pp. 597-626). Torino: Giulio Einaudi.

Testa, E. (2014). L'italiano nascosto. Una storia linguistica e culturale. Torino: Giulio Einaudi.

Tomasin, L. (2001). Il volgare e la legge. Storia linguistica del diritto veneziano (secoli XIII-XVIII). Padova: Esedra.

Ugolini, F. (1859). Vocabolario di parole e modi errati che sono comunemente in uso. Napoli: Stabilimento Tipografico di Federico Vitale.

\section{ON A 1770 RAPE LAWSUIT: GRAMMATICAL NORM, USE AND TEXTUAL INFLUENCES IN SOME APULIAN BUREAUCRATIC}

DOCUMENTS

\section{Summary}

This paper is based on the morphological, syntactic and textual analysis of a lawsuit filed in 1770 for a rape committed in Monteleone, in the province of Foggia. The text properly represents some of the most relevant linguistic characteristics of a broader corpus, which consists in thirty trial files collected in the "serie IX" of the "Dogana delle pecore" fonds belonging to the Provincial Archives. Following the most recent proposals of the linguistic research, the analysis focuses on both the relationship between phenomena, coeval grammars and uses, as well as on the connection between linguistic habits and textual typology. The aim is to point out that the above-mentioned documents are marked by the co-existence between the forms and the uses of the bureaucratic Italian, and those of the italiano dei semicolti.

Keywords: bureaucratic Italian, italiano dei semicolti, linguistic uses and textual typology, linguistic uses in the $18^{\text {th }}$ century, rape lawsuit, Regia Dogana della mena delle pecore. 